\title{
The effects of transition probability of reinforcement in intermittent reinforcement schedules in human eyelid conditioning'
}

J. DAVID HIGGINS, DEPARTMENT OF PSYCHOLOGY, PENNSYLVANIA STATE UNIVERSITY, University Park, Pa. 16803, AND WILLIAM F. PROKASY, DEPARTMENT OF PSYCHOLOGY, UNIVERSITY OF UTAH, Salt Lake City, Utah 84112

Exposing Ss to 50\% intermittent reinforcement schedules in which the likelihood of a reinforced (nonreinforced) trial following a reinforced (nonreinforced) trial differed from the .5 that is typically employed yielded sequential behavior which differed from that obtained when the transition probability equals.5. The new finding was that when the transition probability is high, the sequential behavior did not conform to what would be expected from a linear-operator model.

When exposed to a $50 \%$ reinforcement schedule in which the conditional probability (hereafter called "transition probability") of a reinforced (nonreinforced) trial following a reinforced (nonreinforced) trial equals .5, human Ss exhibit sequential behavior that corresponds to what would be expected from a linear-operator model (Bush \& Mosteller, 1951). That is, response probability increases with successive reinforcements, and decreases with successive nonreinforcements (Prokasy, Carlton, \& Higgins, 1967). Similar incremental-decremental behavior is observed even when the transition probability does not equal the reinforcement ratio. Specifically, if the $50 \%$ schedule is biased toward runs of reinforcements and nonreinforcements, the sequential behavior was as anticipated; however the changes in probability as a function of the sequences were more marked than those obtained when the transition probability equalled the reinforcement ratio. Also, when the intermittent reinforcement schedule was biased toward alternation between reinforced and nonreinforced trials, response probability as a function of successive reinforcements and nonreinforcements was not in any way reliably different from that obtained when the transition probability equaled the reinforcement ratio. The purpose of this experiment was to extend the above findings by employing the same three $50 \%$ intermittent reinforcement schedules at different interstimulus intervals (ISIs). Method

The method of transducing and recording eyelid responses, as well as other methodological detail, is reported elsewhere (Higgins, 1968; Prokasy et al, 1967). The CS was a change in brightness of a 7.5-W bulb, the UCS was a 50-msec airpuff of sufficient intensity to support a $150-\mathrm{mm}$ column of $\mathrm{Hg}$, and CS duration equalled ISI. There were three levels of ISI: 360,500 , and $800 \mathrm{msec}$, and these were orthogonal to three levels of $50 \%$ reinforcement schedule. The schedules differed only in the transition probability. In schedule $2 \mathrm{R}$, this probability was .2 ; in schedule $5 \mathrm{R}$ it was .5 ; and in schedule $8 R$ it was 8 . It should be noted that schedule $5 R$ is what is typically referred to as a "random" schedule. Ss were 270 students from introductory psychology courses who were assigned unsystematically until there were $30 \mathrm{Ss}$ in each of the 9 treatment combinations. Ss were given 12 adaptation trials 6 CS and 6 UCS trials in random order) followed by 300 conditioning trials. The average intertrial interval was $10 \mathrm{sec}$.

Results

Aside from increases in response probability across trial blocks, the only reliable effects observed in the study were the effects of sequences of reinforced and nonreinforced trials, and these results are plotted in Fig. 1. The outcome for Groups $8 R$ and $5 R$ reproduce earlier findings (Prokasy et al, 1967): response probability increases following a reinforced trial and decreases following a nonreinforced trial. Also, the increments and decrements were larger in Group 8R than in Group 5R. The behavior of Group $2 \mathrm{R}$, however, was different. Following a transition to the first reinforcement in a sequence there is a decrease in response probability on the next trial, and following the first nonreinforcement in a sequence there is an increase in probability. With additional reinforcements and nonreinforcements, increments and decrements, respectively, were obtained and are consistent with the outcomes in Groups $8 R$ and $5 R$.

This initial reversal in the sequential behavior of Group $2 R$ was examined in more detail. Table 1 provides response probability for the first two points of the curves of Group $2 R$, but they are additionally broken down into two blocks of 150 trials each. The reversal for the first trial block is reliable, the interaction between Sequence and Event is reliable, $F(1,87)=4.01$. The Sequence by Event interaction is not reliable for the second trial block $F(1,87)$ $=.78$.

Discussion

As shown by Prokasy et al (1967) transition probability does not affect overall gross levels of responding, but it does markedly affect the fine-grain structure of the data. When the transition probability is low, the increment in response probability with successive reinforcements and nonreinforcements is large. The new observation in this study is that when the transition probability is high, the change in response probability with the first in a sequence of reinforcements or nonreinforcements is not in the direction which would be expected from a linear-operator model

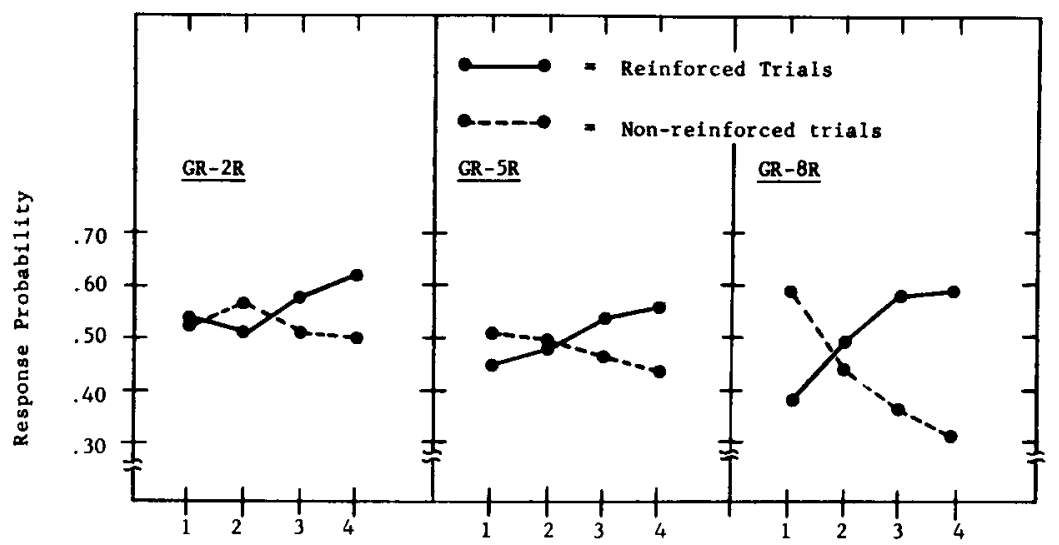

NUMBER OF SUCCESSIVE REINFORCED OR NON-REINFORCED TRIALS
Fig. 1. Response probability as a function of sequences of reinforced or nonreinforced trials. 
Table 1

Response Probability of Group 2R for the First Two Points of the Sequential Curves of Reinforcement and Nonreinforcement

\begin{tabular}{lccc} 
Event on Trial N & Trials & Trial N & Trial N + 1 \\
Reinforcement & $1-150$ & .48 & .46 \\
& $151-300$ & .59 & .59 \\
Nonreinforcement & $1-150$ & .46 & .49 \\
& $151-300$ & .59 & .61 \\
\hline
\end{tabular}

(Bush \& Mosteller, 1951). It can be conluded that $50 \%$ reinforcement schedules with high to medium transition probabilities result in conditioning performance that is not incompatible with a linear-operator model, but that with a low transition probability a model with additional, or different, assumptions is required.

\section{REFERENCES}

BUSH, R. R., \& MOSTELLER, F. A model for stimulus generalization and discrimination. Psydhol Rev., 1951, 58, 413-423.

HIGGINS, J. D. Effects of probabilistic reinforcement schedules and interstimulus interval on the conditioned eyelid response. Unpublished Master's Thesis, Pennsylvania State University, 1968.

PROKASY, W. F., CARLTON, R. A., \& HIGGINS, J. D. Effects of nonrandom intermittent reinforæment schedules in human eyelid conditioning. J. exp. Psychol, 1967, 74, 282-288.

NOTE

1. This study was supported by NSF Grant GB-3875 to William F. Prokasy. This report is based on a study submitted by J. David Higgins to the Pennsylvania State University as partial fulfillment for the requirements of the M.S. degree. 